\title{
The Design of Commercial Signboard Fonts in Shenyang to Establish Urban Visual Orders
}

\author{
Dong Yan $^{1^{*}}$, Wang $\mathrm{Li}^{2}$, Wang YingZhi ${ }^{3}$ \\ ${ }^{1}$ Dalian University of Science and Technology, Dalian, Liaoning, 116052, China \\ ${ }^{2}$ Dalian University of Science and Technology, Dalian, Liaoning, 116052, China \\ ${ }^{3}$ Dalian University of Science and Technology, Dalian, Liaoning, 116052, China
}

\begin{abstract}
Based on the idea of establishing urban visual orders, this paper aims to explore the demonstration form of Shenyang commercial signboard font designs under the influence of the visual order establishment and to handle problems currently existing on streets of Shenyang and major cities, including the chaos in words on commercial signboards that exerts an influence on the visual order. Method The innovative design plan is finally completed via on-site theoretical and survey research. Conclusion The design of commercial signboard fonts is an important element exerting an influence on the overall visual order of one street and even of a city. Two necessary methods of improving urban and regional visual environments are as follows: to fully consider the coordination between commercial signboard fonts and corresponding surrounding environments; and to well control such variables of commercial signboard fonts as forms, colors and materials.
\end{abstract}

\section{Introduction}

A city is a combination of regional cultures and civilizations. Shenyang is a key regional city that is the most representative in Northeast China. As a window of urban civilizations, main streets, store signs and advertising logos are the main carriers of building urban visual images. However, many individual commerce operators, in order to attract customers, often make their stores more dazzling and outstanding by using nonstandardized traditional Chinese characters and variant Chinese characters, which incurs oversize fonts and mismatches between font colors and overall stores. Hence, a relatively big negative effect is exerted on the urban visual order.

As an end-product featuring one era, commercial signboard fonts act as a refractor of aesthetic orientation and spiritual pursuits of local residents. Traditional printed fonts are adopted mainly in some remote and small-scale stores, where changes following the era fail to occur. That is not in line with Shenyang's current overall development level and urban visual image.

\section{Commercial Signboard Situations and Visual Order in Shenyang}

\subsection{Relationship between Urban Visual Order and Commercial Signboard}

Signboard designs of one city firstly impress tourists. With various shapes and colors, signboards provide different feelings for our visual nerves and psychological experience. Population expansion will oppress the urban space and lower life qualities. Similarly, every signboard is scrambling to attract our eyes. This rapid and chaotic development must exert an influence on the overall space visual relationship and lower the visual order sense of a city. Therefore, signboard designs are closely relevant to urban orders and visual images. Every signboard bears written information, but a few attention is paid to commercial signboard font designs. Many stores fail to choose independently designed fonts or select a font suitable for store architecture styles. Hence, definite ideas are scarce in signboard sizes, colors and technique selections.

\subsection{F The Status Quo of Utilizing Commercial Signboard Fonts in Shenyang}

The demonstration of the urban visual order on streets certainly involves commercial signboard designs with different sizes and types. Signboard fonts with suitable sizes and types not only convey necessary visual information to consumers, but also improve the visual order on streets. Table 1 shows the signboard font utilization situations on one certain street in Shenyang on the basis of field surveys.

\footnotetext{
"Corresponding author's e-mail: 274261074@qq.com
} 
Table 1. Table 1 Morphology \& Type of Commercial Signboard Fonts in Shenyang

\begin{tabular}{l|l|l}
\hline $\begin{array}{l}\text { Commercial } \\
\text { Classification }\end{array}$ & \multicolumn{1}{c}{ Font Type } & \multicolumn{1}{c}{ Morphological Feature } \\
\hline $\begin{array}{l}\text { Stonvenience } \\
\text { Barbershop }\end{array}$ & $\begin{array}{l}\text { Mostly in Bold, Partly in independently } \\
\text { designed fonts }\end{array}$ & Oversize fonts, Random forms \\
\hline Hardware Store & $\begin{array}{l}\text { Mostly in Bold, Partly in Running \& } \\
\text { Regular Scripts }\end{array}$ & $\begin{array}{l}\text { Gorgeous and unreal Image, Poor } \\
\text { Identification }\end{array}$ \\
\hline Others & $\begin{array}{l}\text { Sost of fonts used by small individual commercial tenants bear no features and are } \\
\text { relatively easy }\end{array}$ \\
\hline Conclusion & $\begin{array}{l}\text { Apart from stores of independent brands, most of stores face an incorrect utilization of } \\
\text { various signboard fonts }\end{array}$ \\
\hline
\end{tabular}

The survey shows that although most of commercial signboard fonts on the Shenyang commercial streets are diversified, there are abuses and disorderly uses of fonts. Apart from uniform urban planning or brand sponsorship, patterned or immobilization problems lie in font uses. Printed fonts in bold, or regular scripts are largely adopted on the uniformly planned streets, while the handwritten, deformed art letting and printed fonts are adopted in a stagger manner at the unplanned regions, which is more random and irregular.

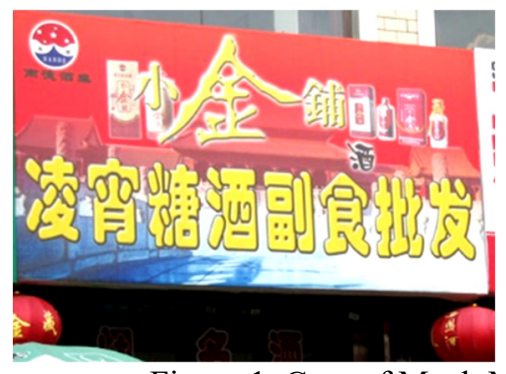

Figure 1. Case of Much More Words on Signboards.

In addition, many commercial tenants, especially small commercial stores, neglect professional features of signboard designs in order to lower manufacturing costs and make fonts bigger \& striking, which incurs a disorder in the design relationship between fonts and signboards (such as font selection, color utilization, format design and technique demonstration). Hence, a
The primary function of signboard words is to convey information to the general public. However, some signboards want to convey much more information, which makes such signboards filled with various words. That will generate not only a strong sense of oppression in visions, but also mismatches in operation ideas. Primary and secondary relations among signboard contents may also be confused. The single addition neglects consumers' visual feelings and even exerts a negative influence on visual orders of surrounding environments and streets. Please look at Figure 1.

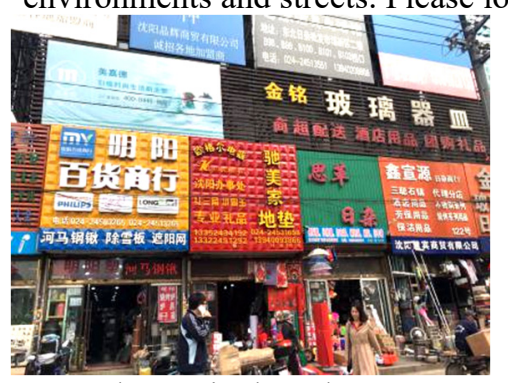

strong sense of disorder exists on many streets. The sizes, formats, colors and techniques of some signboards fail to be designed according to the overall architecture style of streets, which incurs an abrupt comparison between signboards and architectures and brings a kind of visual and psychological pressure. Please look at Figure 2.
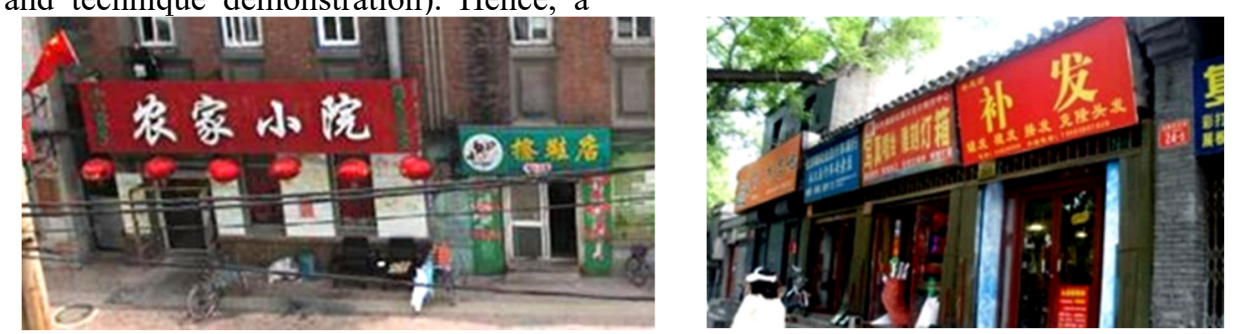

Figure 2. Case of Contradictions between Signboard Fonts and Surrounding Environments \& Architecture Styles 


\section{To Promote the Establishment of Urban Visual Orders via Commercial Signboard Font Designs}

\subsection{To Establish Corresponding Visual Orders according to Different Regional Cultures}

Relatively speaking, the regional culture in Northeast China is passive and acceptance-oriented, the changes of which largely depend on exchanges with foreign cultures. That's why the regional culture in Shenyang is full of complexity. The current cultural features in Shenyang integrate various elements, including Manchu, the Republic of China (1912-1949), revolutions and industries. Therefore, under the urban cultural background with complex regional elements, commercial signboard designs shall exert a positive influence in order to respond to urban cultures and create distinctive urban features via emotional font designs. Signboard fonts that are incompatible with contexts shall be avoided on special culture streets, otherwise an inappropriate sense may be incurred.

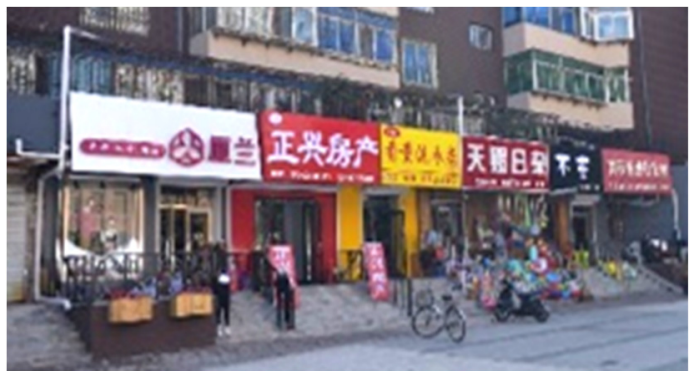

Figure 3. Commercial Signboards on the Bagua Street

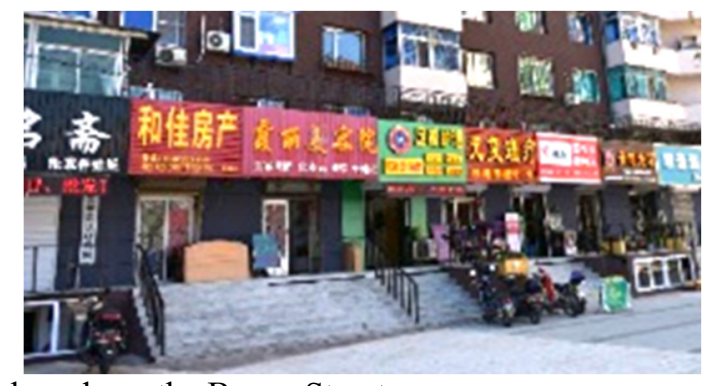

the Bagua Street shall highlight its custom elements during the Republic of China (1912-1949). Please look at Figure 4. Through the "Republic-of-China Style" design, commercial signboards on the Bagua Street integrate with old architectures nearby, which creates a unified sense of visual orders. Therefore, the era sense and historical cultures of the Bagua Street can be truly appreciated. cultural features of the Bagua Street, the design positioning of commercial signboards on the street shall be in line with its whole reconstruction subject. Namely,

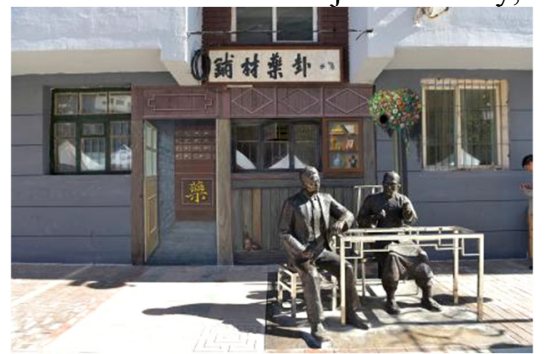

Figure 4. The "Republic-of-China Style" Mura

\section{The Design of Signboard Fonts to Create Special Commercial Cultures of the Bagua Street}

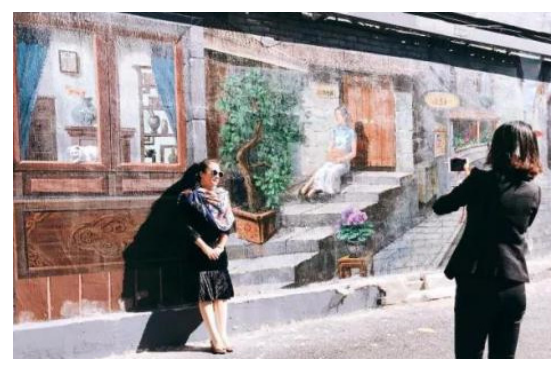

The font style of commercial signboards that is in line with the visual order of the Bagua Street is dated from the early period of the Republic of China (1912-1949), the construction era of the Bagua Street. According to investigations and research, the structures of signboard fonts largely adopted during the period of the Republic of China are slimmer and longer than normal ones. Concretely speaking, the middle part of such structures is

\subsection{Font Style}


tight, the upper and bottom parts are open and the left and right parts are constrictive. To stretch fonts at the visual level provides a slender and straight sense. Please

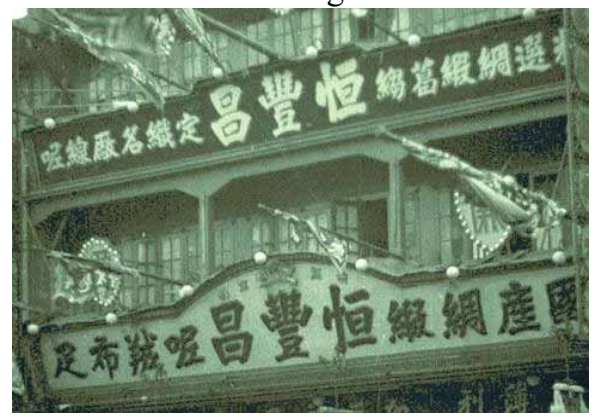

Figure 5. Signboard Font Style during the "

\subsection{Innovations in Morphological Features}

In combination with the font style during the Republicof-China period (1912-1949), the author decides to design a kind of font by regarding the Bangshu in the Qing Dynasty as the framework. Please look at Figure 6. In order to make fonts creative and full of visual shocks, some features of the Song Typeface are added into the look at Figure 5. Therefore, the font designs of the Bagua Street should consider the "Republic-of-China Style" as the pursuit.

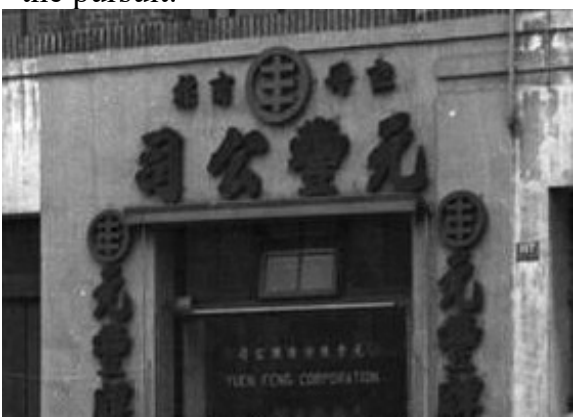

"Republic-of-China" Period (1912-1949)

Regular Script basis of Bangshu, including the first stroke, the final stroke and 顿笔 (as shown in Figure 6). This kind of font is in line with the sense of historical eras, integrates vigorous and powerful features of Bangshu and Regular Script in handwritten forms with the Song Typeface's unique serifs and decoration angles, and pursues detailed features of fonts that are easy, legible and suitable for commercial promotion.

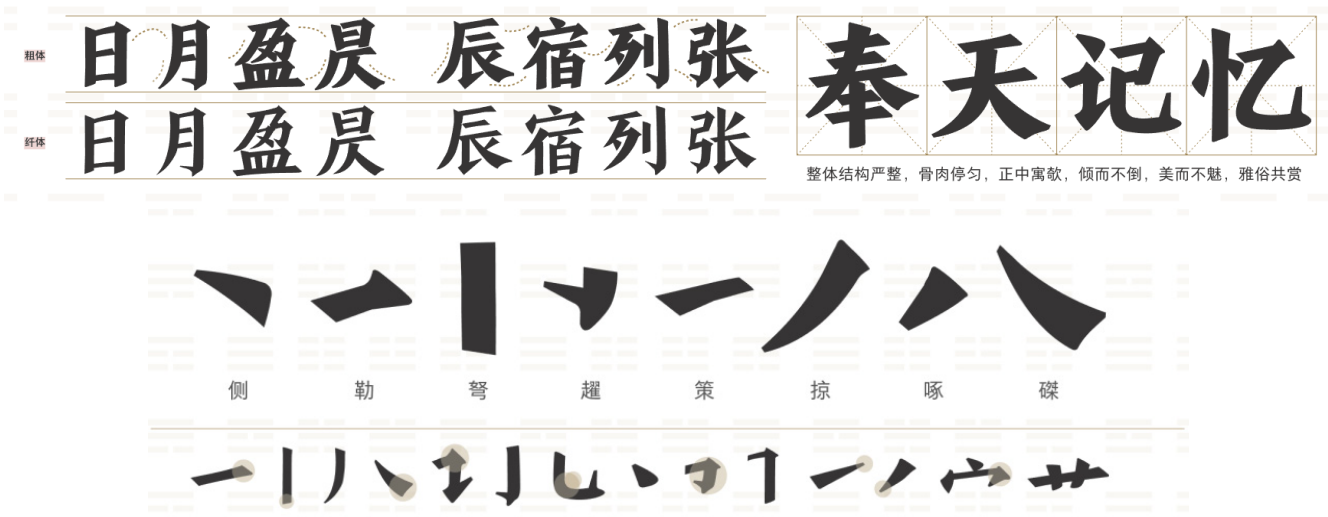

Figure 6. Design Features of Regular-Script Commercial Signboards during the Republic-of-China Period(1912-1949)

\subsection{Utilization Standards and Situations}

After font designs are finished, corresponding utilization conditions are standardized in order to restrain variables, such as font formats, sizes and colors. Based on measurement and calculation, the author believes that there must be an inviolable area with a certain size on

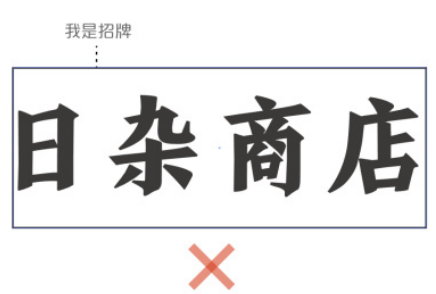
signboards (as shown in Figure 7) and provides concrete contents as follows: various mixed font collocation cases; color collocation standards; and different formats designated according to store features. As to utilization situations, this font is suitable for commercial streets or tenants that are in pursuit of the era sense of the Republic-of-China Period (1912-1949).

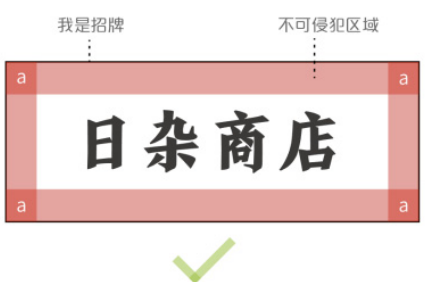

Figure 7. Utilization Standards for Regular-Script Commercial Signboards during the Republic-of-China Period (19121949)

\subsection{Effect Evaluation Forecast}

This kind of font regards the Bangshu as its framework and integrates the Song Typeface's shapes, which is extremely similar to handwritten commercial signboard fonts during the Republic-of-China period and also makes a large number of changes. Therefore, the historical and cultural backgrounds of commercial stores can be fully demonstrated, which will create a sense of immersion at the visual and psychological levels. If this 
kind of font can be utilized on the Bagua Street in accordance with some standards, current problems of chaotic visual orders caused by commercial signboard fonts will be solved to some extent. At the same time, we should design more types of fonts similar to such one, which can provide commercial tenants with more choices in signboard fonts.

\section{Conclusions}

The establishment of urban visual orders is to commercial signboard fonts what one wall is to one brick. The neglect of any brick can exert an influence on a windowless wall. Nowadays, many cities fail to attach enough importance to commercial signboard font designs, which makes them all the same. Based on this phenomenon, a fact should be highlighted that designs, management and plannings of commercial signboard fonts on urban streets are strengthened, which is of greatest significance equivalent to strengthening urban landscaping.

\section{References}

1. Ma Quan. A Joint Establishment of New Visual Orders in Cities [J]. Admen, 2010, (08):202-203.

2. Chai Zhi. A Study on the Aesthetic Order in Visual Communication Design [J]. Journal of Yangtze University (Social Science Edition), 2013, 36 (10):201-203.

3. Li Chuanlin. The Research and Redesign of Commercial Signboard Words during the Republic of China (1912-1949) [D]. Nanjing University of the Arts, 2016.

4. Shao Ting, Zhang Minyan. The Sign Design of Enhancing the City's Identity [J]. Design, 2015, (05):35-36.

5. Tang Yuting. Commercial Shop Signs under the Design of New Technologies [D]. Henan Normal University, 2014.

6. Liu Xin. On the Sense of Order Existing in Visual Communication Designs [D]. Jiangxi Normal University, 2013. 